\title{
A HIGH-RESOLUTION STUDY OF LOCAL INTERSTELLAR SODIUM
}

\author{
A. Ardeberg, H. Lindgren, E. Maurice \\ European Southern Observatory \\ Lund and Marseille Observatories
}

\section{ABSTRACT}

A high-resolution spectroscopic investigation has been made of interstellar lines of sodium. From identifications of $D_{1}$ and $D_{2}$ line components concentrations of interstellar sodium gas have been studied. Some preliminary data are provided on the spatial distribution of stronger sodium concentrations.

\section{INTRODUCTION}

For investigations of stronger concentrations of interstellar sodium gas spectroscopic identification of line components is a standard method. For the weak line components to be expected from local gas, identifications are more difficult than normally due to influence of stellar and atmospheric lines and, in case of more distant background stars, also due to blending by stronger sodium components from richer concentrations of interstellar sodium along the line of sight. Finally, the signal-to-noise ratios necessary for identification of faint lines require special observing techniques.

\section{OBSERVATIONS}

With the ESO Coudé Echelle Spectrometer at a resolution of $10^{5}$ we have observed the interstellar lines of sodium at 5890/5896 \&. A total of around 250 observations have been made for 200 stars. With the Reticon array detector very high signal-to-noise ratios can be obtained. This means that at least for brighter stars we can avoid line-identification difficulties due to the limitations in the observing technique.

\section{IDENTIFICATION OF CONCENTRATIONS OF INTERSTELLAR SODIUM}

There are several features aiding us in our survey for faint line components of interstellar sodium. First, our spectral resolution is very high. Second, the signal-to-noise ratio of our spectrograms is very favourable, for brighter stars often well above $10^{3}$. Third, the fact that both $D_{1}$ and $D_{2}$ are included is of high importance.

For a first analysis we have inspected our spectrograms visually and identified the stronger line components of interstellar sodium gas. Especially 
for the line components resulting from local gas concentrations this provides only a lower limit to the number of interstellar sodium components really available.

Following the visual survey, a more detailed analysis is now in progress. A most important feature in this analysis is a careful mapping of the lines of atmospheric water vapour present in the spectral range of interest. From spectrograms of early-type stars and without interstellar sodium lines the relative atmospheric water-vapour spectrum is obtained. Measurements of the strengths of selected water-vapour lines in the programme spectrograms then transforms the relative atmospheric water-vapour spectrum into an absolute one. Correction for these lines provides programme spectrograms of considerable purity. Also very faint sodium-1ine components can then be identified.

For our detailed analysis of lines of interstellar sodium detailed account has to be taken also of the influence of stellar lines. Mapping of such lines for all spectral classes concerned is therefore very important.

Mutual blending of sodium lines from interstellar gas is another problem which needs careful attention. The problem is especially serious when distant stars are used and the line of sight crosses more massive sodium clouds outside the local solar neighbourhood. Spatial line mapping is the most direct approach. Still, blending can be rather serious, demanding facilities for powerful disentangling procedures. A special deconvolution programme has been developed for our purpose. The use of this programme is greatly facilitated by the presence of the two sets of sodium lines.

\section{DISTANCES OF STARS}

From available spectral classifications on the MK system and from UBV photometry distance moduli, corrected for influence of interstellar absorption, have been determined. They should be of an accuracy relevant for our present preliminary analysis.

\section{PRELIMINARY RESULTS}

In Figures 1-3 we present our preliminary estimate of the spatial distribution of interstellar sodium gas. For three intervals in galactic latitude we have noted the number of (stronger) interstellar lines in plots of distance modulus versus galactic longitude.

As could be expected, the stars most nearby do not display many stronger line components of interstellar sodium. However, there are examples of components of significant strength obviously resulting from gas clouds quite close to the sun. It is interesting to note that this is true also for stars situated outside the galactic plane.

The data presented indicate a number of nearby concentrations of interstellar sodium gas. It is our intention to follow this up in two ways. First, detailed analysis of our present spectrograms should provide increased accuracy and resolution for the local interstellar lines. Particularly, 
fainter lines can be studied and thereby more components be identified. Second, more observations are planned for the nearby clouds indicated in the present work as well as for parts of the sky so far less well covered by our observations.

A programme is under way to determine radial velocities for all components of interstellar sodium from the observing material discussed in the present paper. We expect an accuracy of the order of $1 \mathrm{kms}^{-1}$. At the same time, line intensities w11l be measured for all components of interstellar sodium. Combination of radial velocities and line intensities will provide useful data on the distribution and kinematics of local sodium gas. Moreover, it means that much better use can be made also of more distant stars for the study of local gas clouds.

In this presentation we have excluded results from a relatively extensive study of interstellar sodium in the direction of IC 2944. This study clearly indicates at least two local gas components. The agreement with the present work is good.

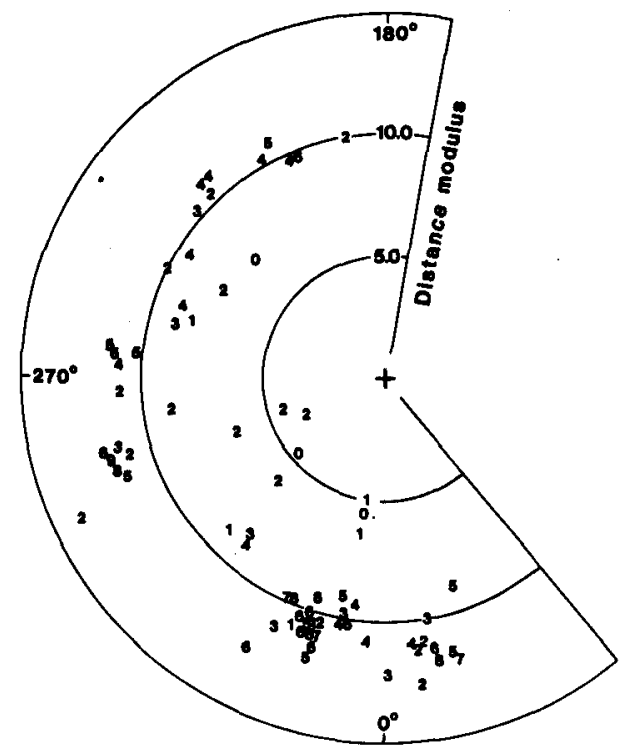

Fig. 1: Our estimated lower limits to the number of interstellar line components given in a diagram with distance modulus versus galactic longitude. Interval in galactic latitude, $b$, is $-5^{\circ}<b<+5^{\circ}$. 


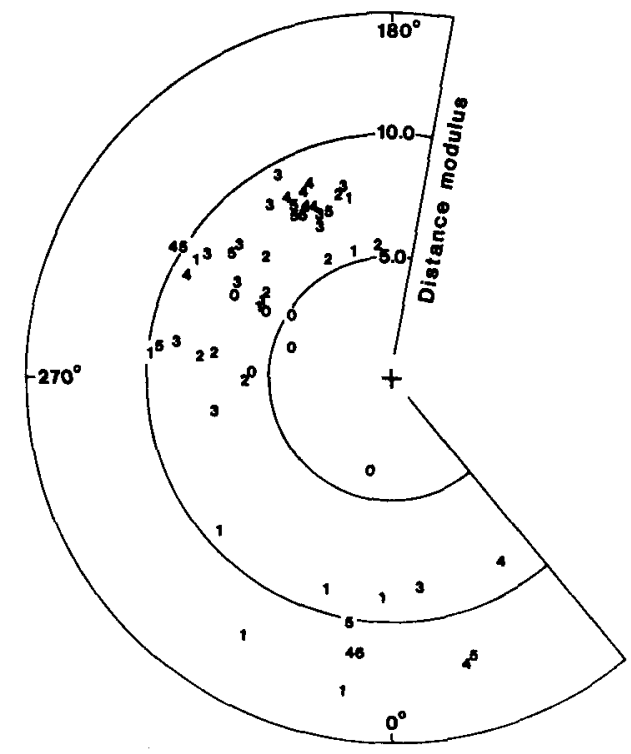

Fig. 2: Same as Figure 1 but for the latitude interval $-25^{\circ}<b<-5^{\circ}$.

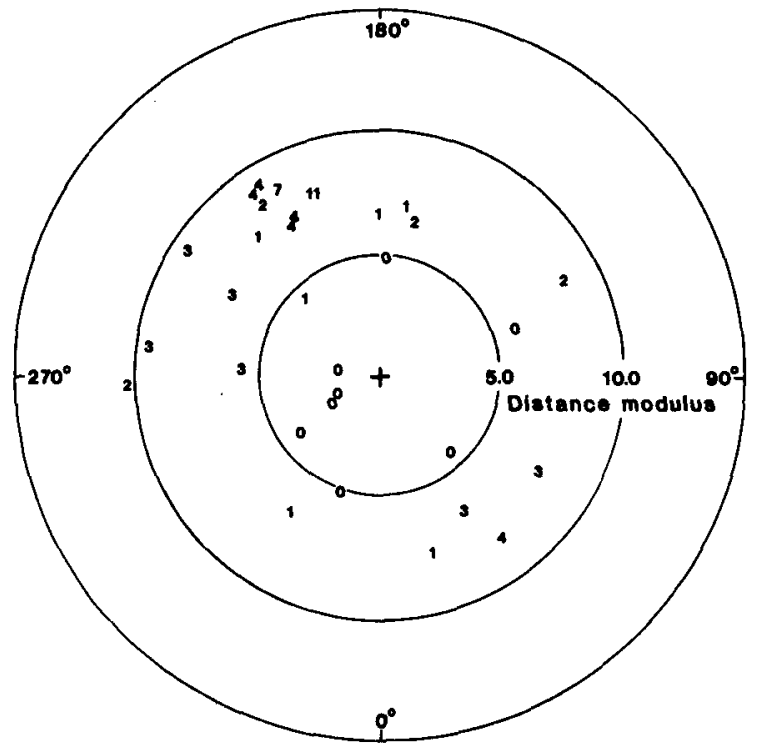

Fig. 3: Same as Figure 1 but for the latitude interval $\mathrm{b}<-25^{\circ}$. 